\title{
BMJ Open Efficacy of metacognitive therapy for prolonged grief disorder: protocol for a randomised controlled trial
}

\author{
Jenine Wenn, Moira O'Connor, Lauren J Breen, Robert T Kane, Clare S Rees
}

To cite: Wenn J,

O'Connor M, Breen LJ, et al. Efficacy of metacognitive therapy for prolonged grief disorder: protocol for a randomised controlled trial. BMJ Open 2015;5:e007221. doi:10.1136/bmjopen-2014007221

- Prepublication history for this paper is available online To view these files please visit the journal online (http://dx.doi.org/10.1136/ bmjopen-2014-007221).

Received 16 November 2014 Revised 28 September 2015 Accepted 16 October 2015

CrossMark

School of Psychology and Speech Pathology, Curtin University, Perth, Western Australia, Australia

Correspondence to Jenine Wenn, jenine.wenn@postgrad.curtin. edu.au

\section{ABSTRACT}

Introduction: Studies of effective psychotherapy for individuals suffering from the effects of prolonged grief disorder (PGD) are scarce. This paper describes the protocol for an evaluation of a metacognitive therapy programme designed specifically for PGD, to reduce the psychological distress and loss of functioning resulting from bereavement.

Methods and analysis: The proposed trial comprises three phases. Phase 1 consists of a review of the literature and semistructured interviews with key members of the target population to inform the development of a metacognitive therapy programme for Prolonged Grief. Phase 2 involves a randomised controlled trial to implement and evaluate the programme. Male and female adults $(\mathrm{N}=34)$ will be randomly assigned to either a wait list or an intervention group. Measures of PGD, anxiety, depression, rumination, metacognitions and quality of life will be taken pretreatment and posttreatment and at the 3-month and 6-month follow-up. The generalised linear mixed model will be used to assess treatment efficacy. Phase 3 will test the social validity of the programme.

Discussion: This study is the first empirical investigation of the efficacy of a targeted metacognitive treatment programme for PGD. A focus on identifying and changing the metacognitive mechanisms underpinning the development and maintenance of prolonged grief is likely to be beneficial to theory and practice.

Ethics: Ethics approval was obtained from Curtin University Human Research Ethics Committee (Approval number HR 41/2013.)

Trial registration number: ACTRN12613001270707.

\section{INTRODUCTION}

It is increasingly argued that a significant minority of bereaved individuals experience a more complicated form of grief called prolonged grief disorder (PGD) following the death of a significant other. ${ }^{1-4}$ Their prolonged grief causes significant social and occupational impairment and is associated with suicidality, poorer health-related quality

\section{Strengths and limitations of this study}

- This is the first randomised controlled trial testing the effectiveness of a targeted metacognitive treatment intervention to reduce prolonged grief disorder symptomatology.

- The study will establish the mechanisms by which the intervention is effective.

- The study will also determine if a short manualised intervention is acceptable for people with prolonged grief disorder.

- If the intervention is not acceptable, this study will identify the factors contributing to a negative outcome.

of life, substance abuse ${ }^{23}$ and a reduced likelihood to seek assistance from mental health services. $^{5} \quad$ These individuals experience separation distress, involving an unrelenting yearning for the deceased, a sense of meaninglessness, and difficulty accepting the loss, all of which remain elevated for 6 months or more following the loss. ${ }^{4-6}$ Individuals experiencing PGD also fail to exercise regularly, withdraw from social activities and neglect their own nutrition. ${ }^{7}$

Grief was historically excluded from diagnostic nosology on the basis that it was an inevitable and natural response to a traumatic event. ${ }^{4}$ However, persistent complex bereavement disorder is a condition for further study in the latest Diagnostic and Statistical Manual of Mental Disorders (DSM) $)^{8}$ and PGD is likely to be included in the forthcoming International Classification of Diseases (ICD). ${ }^{9}$ There is growing empirical evidence supporting the distinction between normal and diagnosable grief reactions and attention has turned to the development of treatments specifically for PGD.

\section{Grief interventions}

The lack of understanding of grief within a clinical framework has been a barrier to effective treatment interventions. ${ }^{10}$ Although the emphasis on 'working through' different 
stages of grief has been challenged in recent years, the idea remains uncritically accepted by many service providers, ${ }^{10}$ and many grief service providers still draw on stage, task and process models. ${ }^{11}{ }^{12}$ Grief interventions for 'normal' grief have not been efficacious and in many cases have even obstructed grief processes. ${ }^{13}$

Interventions based on standard treatments for depression show minimal effects. ${ }^{14}{ }^{15}$ However, treatments tailored to PGD symptoms demonstrate better outcomes. ${ }^{16-18}$ For example, one study ${ }^{17}$ compared cognitive behavioural therapy (CBT; 6 sessions of cognitive restructuring and 6 sessions of exposure therapy) with 12 sessions of supportive counselling (SC). CBT was found to be more effective than SC, and the exposure therapy component of CBT was found to be more beneficial than cognitive restructuring, suggesting that avoidance behaviours may be more central to PGD than the content of thoughts. Similarly, another study ${ }^{18}$ adapted interpersonal psychotherapy for depression (IPT) into a complicated grief treatment (CGT) that targeted depressive symptoms and trauma over 16 sessions. CGT was found to have significantly greater reductions in symptomatology than IPT alone, providing support for the need to tailor treatments to symptomatology. However, only $51 \%$ of the intervention group responded to treatment, suggesting more work is required in this area.

The need for an effective treatment for PGD is further supported by a recent meta-analysis, ${ }^{19}$ which found that interventions targeting complications experienced in grief have only had moderate effect sizes. One of the possible reasons for the moderate effect sizes may be that therapies were not based on a theoretical model underpinning all the symptoms of PGD. The dominant model is the cognitive behavioural model, which suggests difficulty integrating the loss into autobiographical knowledge, negative global beliefs, misconceived grief symptoms and avoidance behaviours underpin the development and maintenance of PGD. ${ }^{20}$ CBT primarily focuses on exposure to traumatic memories, the repetitive recall of events, behavioural activation and restructuring the content of maladaptive thoughts. While maladaptive thoughts (eg, 'I'm not good enough'; 'I'm vulnerable'; 'I'm at threat') are characteristic of depression, anxiety and post-traumatic stress disorder (PTSD), PGD is distinct from these disorders. Although some overlapping in symptomatology occurs with that of depression, anxiety and PTSD, symptoms of separation distress, yearning, disbelief and a sense of meaninglessness are unique to PGD. ${ }^{20}{ }^{21}$ PGD involves preoccupation with mental anguish over the reality of the loss and an inability to consider a future without the deceased. ${ }^{21}$ The metacognitive model would suggest that treatment effectiveness may be improved by shifting the focus away from reappraisal of the content of thoughts to focusing on modifying unhelpful metacognitive processes understood to maintain distress. ${ }^{22}$ The death of a loved one comprises many realistic thoughts involving the events surrounding the death, shock that the loved one will not be returning and the financial stressors now faced. Therefore, addressing how bereaved individuals process loss-related thoughts may be more effective than addressing what they think.

Studies have identified that some bereaved individuals ruminate over memories of the deceased in an effort to 'make sense' of the death, ${ }^{23}$ or to 'properly mourn' or to maintain bonds with the deceased. ${ }^{10} \quad 24 \quad 25$ Importantly, studies have clearly demonstrated that rumination intensifies grief symptoms. ${ }^{26}{ }^{27}$ Rumination has been shown to be an anxious avoidant coping strategy, whereby events in the past and counterfactual thoughts (eg, 'what if I'd stayed home that day') are rehashed by the bereaved to avoid emotions and thoughts related to the reality of the loss. ${ }^{28}{ }^{29}$ Rumination, postevent processing and worry have a common underlying construct called repetitive negative thinking, which has recently been identified as a transdiagnostic process. ${ }^{30-32}$ Repetitive negative thinking about issues related to past experiences or worries about the future ${ }^{30}$ keeps attention fixed on distressing information (eg, the injustice and consequences of the loss), which impairs the chance of developing coping strategies, promotes maladaptive behaviours, drives away social support and perpetuates depressive symptoms. ${ }^{33} 34$ A recent study ${ }^{35}$ found individuals with low social support ruminated more, causing higher levels of PGD symptoms and lower levels of positive mood. For grief treatment to be effective, it is important that techniques are grounded within an information processing framework that targets the way distressing information is processed, to prevent people developing maladaptive coping processes (eg, rumination and worry). The metacognitive model posits metacognitions give rise to maladaptive patterns of thinking. ${ }^{36}$ Therefore, a treatment based on a metacognitive model may be better suited to PGD, as this model targets the way information is processed rather than focusing on the content of thoughts.

\section{The metacognitive model of psychological disorder}

The metacognitive model suggests psychological disorders result from repetitive negative thinking over a perceived belief (eg, the world is dangerous), creating an attentional bias due to a constant focus on and monitoring of this belief. $^{22}$ Therefore, individuals with a high level of repetitive negative thinking are less likely to develop coping strategies and more likely to develop a cognitive attentional syndrome. The cognitive attentional syndrome, comprising worry/rumination, threat monitoring and maladaptive coping behaviours, has been found to intensify and prolong psychological distress. ${ }^{36}$ Metacognitive beliefs about the function of these coping processes maintain the cognitive attentional syndrome. ${ }^{36}$ Given that rumination is a key factor identified in the development and maintenance of PGD, a metacognitive model of psychological disorder would suggest that modification of this dysfunctional thinking style would be essential in treating this condition. 
Metacognitive therapy (MCT) alters a dysfunctional thinking style by helping individuals understand their 'thoughts about thoughts' and regulate unhelpful thoughts and feelings. It uses detached mindfulness to educate people to be 'mindful' of thoughts, emotions, beliefs and memories without exclusively focusing on them, and to help people see themselves as separate to their thoughts. ${ }^{36}$ MCT deals with intrusive symptoms, allowing individuals to shift from self-processing by interrupting negative thoughts and gaining a flexible non-repetitive thinking style. ${ }^{36}$ This model complements the dominant model of grief which proposes that the moving between loss and restoration domains allows the grieving individual to gradually accommodate the loss. ${ }^{37}$

\section{Efficacy of metacognitive treatment}

Efficacy of MCT has been demonstrated in studies of major depressive disorder ${ }^{38-40}$ obsessive-compulsive disorder; ${ }^{41-43}$ social phobia; ${ }^{44}{ }^{45}$ generalised anxiety disorder $^{46}{ }^{47}$ and PTSD. ${ }^{48-50}$ Wells et $a l^{40}$ evaluated the effectiveness of eight sessions of MCT with a sample of 12 patients with treatment-resistant recurrent Major Depressive Disorder and found large and clinically significant reductions in attentional control, rumination, worry and metacognitive beliefs that were maintained at the 6-month and 12-month follow-ups. The 12-month follow-up revealed that $80 \%$ of patients had recovered; suggesting MCT is an effective treatment for patients non-responsive to antidepressant medication and previous psychological treatments. Moreover, the effectiveness of MCT for chronic PTSD was recently supported in a controlled trial ${ }^{48}$ with 20 patients revealing significant reductions in PTSD symptoms, anxiety and depression in the MCT group at post-test and at the 3-month and 6-month follow-up, with recovery rates of $60-80 \%$ maintained at the 6-month follow-up. These studies demonstrate that MCT is effective in helping patients respond to intrusive and dysfunctional thoughts. Also, excellent outcomes with MCT have been achieved in as little as six sessions, indicating that this approach may offer a brief and cost-effective alternative to the treatment of mental health difficulties. As such, there is potential value in the evaluation of this type of intervention for PGD.

\section{Aims of the MCT trial}

The aims of this study are to: (1) document the types of metacognitive beliefs characteristic of those presenting with PGD and use the information gleaned to guide the development of an MCT programme specifically for bereaved people; (2) investigate the efficacy of this metacognitive grief therapy (MCGT) in reducing psychological distress by conducting a randomised controlled trial; (3) evaluate the integrity of programme delivery and treatment adherence; and (4) determine the appropriateness and acceptability of the MCGT programme for individuals with PGD.

\section{Hypotheses}

1. The intervention group will report significantly greater pre-post decreases in PGD symptomatology, metacognitions, rumination, depression, anxiety and stress, compared to the wait-list group.

2. The intervention group will report a significantly greater pre-post increase in quality of life, compared to the wait-list group.

3. For the intervention group, post-intervention changes in PGD symptomatology, metacognitions, rumination, depression, anxiety, stress and quality of life will be maintained at the 3-month and 6-month follow-up.

4. Symptom change in the intervention group will be mediated by reductions in metacognition and rumination scores.

\section{METHODS \\ Phase 1}

\section{MCGT development}

The cognitive processes that control, monitor and appraise thinking need to be specifically targeted to treat PGD successfully. ${ }^{36}$ Therefore, we needed to explore the metacognitions involved in PGD to ascertain if the metacognitive model was applicable to PGD and to find clinically relevant examples to incorporate into the intervention that bereaved people could personally relate to. A sequential methodological approach was used to develop the MCGT treatment protocol. Step 1 involved a review of the literature to determine the domains known to engage coping processes that disrupt the grieving process (eg, cognitions, emotions, behaviours, etc). This information was used to inform the interview questions for step 2. Step 2 involved semistructured interviews with psychiatrists/psychologists and people with elevated PGD symptomatology to extract the metacognitive beliefs driving maladaptive coping processes within each domain. Step 3 used the information gleaned to adapt 'Metacognitive Therapy for Anxiety and Depression ${ }^{36}$ into a MCGT treatment that directly targets the metacognitive beliefs involved in PGD.

Design: A qualitative research design using semistructured interviews was adopted to provide some topic consistency, while allowing each interview to be adapted to each informant.

Participants: A small sample was considered sufficient ${ }^{51}$ for the information required to refine the existing MCT programme by Wells ${ }^{36}$ into a programme specifically for PGD. Thus, psychiatrists/psychologists $(\mathrm{N}=6)$ willing to share their knowledge and experience were purposively recruited in order to understand the key metacognitions pertaining to PGD. The findings were used iteratively to refine the interview questions for participants with PGD. In investigating people's experiences and cognitive processes, hearing the voices of people with PGD was of utmost importance. Bereaved adults (N=5) with elevated levels of PGD symptomatology (cut-off score of $\geq 26$ ) and social/occupational/functional 
impairment (determined by the PGD Scale $;^{4}{ }^{52}$ ) were recruited through purposive sampling.

Procedure: Participants were interviewed (for approximately $60 \mathrm{~min}$ ) using an interview schedule comprising open-ended questions developed to extract metacognitive beliefs across the domains derived from step 1 . The thoughts from each domain that triggered maladaptive emotions or behavioural responses were explored (eg, by asking what was driving that thought, emotion or behaviour) to find the metacognitions associated with them. Given the complexities and sensitivities of interviewing bereft people, it was necessary to utilise a person-centred method of interviewing so that the participants conveyed their experiences from their own perspectives. The interviewer engaged the participant by asking questions such as: "Can you give me an example of things you do to feel closer to, to remember, or in remembrance of (name)?"... "Why do you do this?"... "What's positive about that?"... "What's negative about that?" The interviews continued until saturation, as defined by no new metacognitions arising. Participants were compensated for their time with a gift voucher. The interviews were audio recorded and transcribed verbatim.

Analysis: The analysis process incorporated constant comparison, which is the continual comparison of data, within and between transcripts, so that similarities and differences are revealed. ${ }^{53}$ This method was used to generate the identification and description of metacognitions involved in PGD to inform the examples used in the intervention.

\section{Intervention content}

The MCGT programme was developed as a modified version of MCT, which targets the metacognitive beliefs guiding maladaptive coping strategies, and eliminates worry/rumination and fixed attention, which in turn allows the metacognitive flexibility required for normal cognitions to occur. ${ }^{36}$ MCT comprises three components: engagement in therapy, MCT and maintenance. The MCGT programme was produced by modifying each of these components, manualised in 'Metacognitive Therapy for Anxiety and Depression' (see table 1; contact first author for details). Treatment manualisation is important in evaluating the effects of a specific treatment, as it allows treatment standardisation and fidelity assessment and an operational definition of the treatment. ${ }^{54}$ These tailored face-to-face sessions (involving 10 or fewer participants) will run for 2 hours once a week over 6 weeks. Two hours per session is thought to assist participants to feel engaged in the group process. ${ }^{55}$ Each group session will be facilitated by the first author and a co-facilitator (both clinical psychology trainees) and will be held at the Curtin University Adult Psychology Clinic or a more geographically appropriate community centre, with refreshments provided. Participants will be asked to complete homework activities between sessions.
Table 1 Outline of 'Metacognitive Grief Therapy'

\section{Session Content}

$1 \quad$ Normalise grief symptoms

Formulate metacognitive model of grief Enhance awareness of metacognitions Introduce metacognition self-monitoring

2 Explore the link between metacognitions, emotions and behaviour

Changing unhelpful metacognitions into helpful metacognitions

Introduce detached mindfulness

Introduce rumination/worry postponement

3 Detached mindfulness practice

Introduce attention training

Challenge positive metacognitive beliefs about rumination/worry

$4 \quad$ Attention training practice

Challenge uncontrollability metacognitions and positive beliefs about rumination

Explore metacognitive beliefs about avoidant coping

Challenge unhelpful metacognitive beliefs about pleasant activity scheduling

5 Detached mindfulness and attention training practice

Programme review

Maintenance planning

6 Detached mindfulness and attention training practice

Relapse prevention

\section{Phase 2}

\section{Participants}

Participants will be bereaved individuals aged 18 years or more who are at least 6 months post the death of a significant other. Participant inclusion criteria are: English speaking; able to provide written informed consent; presence of PGD (determined by PG-13 cut-off score ${ }^{4}$ ); and if taking medication (namely, antidepressants or other mood stabilisers), the medication must be stable for 1 month prior to baseline assessment and dosage and medication must remain the same throughout the treatment period including follow-up. Participant exclusion criteria will be: a high suicidal risk (determined by the Mini International Neuropsychiatric Interview; $\mathrm{MINI}^{56}$ ); current substance abuse or dependence; current other psychological treatment; and a pre-existing psychotic or bipolar disorder or neurological history, according to DSM, 4th Edition, Text Revision (DSM IV-TR) diagnostic criteria, as measured by the MINI. ${ }^{56}$

\section{Sample size}

Hypotheses 1-2 predict Group×Time interactions. Assuming that these interactions are 'moderate to large' $(\mathrm{f}=0.31+)$, then 17 participants per group $(\mathrm{N}=34)$ should give the generalised linear mixed model (GLMM) an $80 \%$ chance of capturing the interaction effects at an $\alpha$-level of 0.01 (allowing for Bonferroni corrections). 
Hypothesis 3 predicts significant therapeutic changes on all outcomes between the pretest and 3-month follow-up and between the pretest and 6-month follow-up. Seventeen participants per group will provide an $80 \%$ chance of capturing 'moderate to large' changes at a Bonferroni-adjusted $\alpha$-level of 0.01 . The mediation effects predicated in hypothesis 4 will be tested by estimating the significance of the mediation pathway with a bootstrapping procedure. Thirty-four participants should sufficiently power this procedure. This sample size was calculated with the aid of $\mathrm{G} \times$ Power $3{ }^{58}$ A moderate to large effect is assumed based on previous MCT intervention studies. ${ }^{4059}$

\section{Recruitment}

Participants from the metropolitan community of Perth, Western Australia will be recruited through advertisements in print media/websites/newsletters/flyers in palliative care centres, bereavement groups, medical and mental health centres, local shopping centre boards, community newspapers, television and radio.

\section{Measures}

\section{Descriptive measures}

The following demographic information will be provided by each participant: age, gender, postcode, general medical history, current psychological treatment, cultural background, relationship with the deceased, and employment status, date and cause of deceased's death.

The Millon Clinical Multiaxial Inventory $\left(\mathrm{MCMI}^{57}\right)$; will be used to describe the clinical personality patterns and severe personality pathology of the population. It is a 175-item true/false self-report questionnaire designed to assess indicators of DSM-V Axis II disorders. The MCMI has shown test-retest reliability coefficients ranging from 0.77 to $0.87 . .^{57} 60$

\section{Diagnostic measures}

The Mini International Neuropsychiatric Interview $\left(\mathrm{MINI}^{56}\right)$; will be used to determine the presence/absence of other possible comorbid disorders. The MINI is a short diagnostic structured interview that provides a diagnosis based on the DSM-V Axis I orICD, Revision 10 (ICD-10) criteria. The suicidality section will be used to assess suicidal risk and ideation. The MINI classifies levels of suicidality into four groups: no suicidal risk, low suicidal risk, moderate suicidal risk and high suicidal risk. It has been shown to have high test-retest reliability (0.76$0.93)$ and validity. ${ }^{61}$

The PGD Scale $\left(\mathrm{PG}-13^{4}\right)$; will be used as the primary measure to determine the diagnostic status and symptom severity of PGD. It is a 13-item self-report questionnaire that assesses PGD symptoms using a five-point Likert scale (0-4) representing increasing levels of symptom severity. One of the two 'separation distress' symptoms and five of the nine 'cognitive, emotional, and behavioural' symptoms are required for diagnosis.
A total score (range: $0-44$ ) is determined by summing the scores. The PG-13 has shown adequate internal consistency (Cronbach's $\alpha=.82$ ) and incremental validity. ${ }^{35}$

\section{Self-report measures}

Core Bereavement Items $\left(\mathrm{CBI}^{62}\right)$; will be used as a general measure of grief severity. The 17 -item questionnaire is scored on a four-point Likert scale ranging from 0 (never) to 3 (continuously), and has three subscales: 'Images \& Thoughts' (7 items), 'Acute Separation' (5 items) and 'Grief' (5 items). A total score can be obtained by summing the subscales ranging from 0 to 51. The scale has shown high internal consistency $(\alpha=.92)$ and validity. ${ }^{62} 63$

Depression Anxiety Stress Scales-21 (DASS-21 ${ }^{64}$ ); will be used to measure symptoms of depression, anxiety and stress. The 21-item questionnaire requires participants to rate symptoms on a four-point Likert scale ranging from 0 (did not apply to me at all) to 3 (applied to me very much, or most of the time). The scale consists of depression, anxiety and stress subscales that can be summed to produce a total distress score. Higher scores indicate higher symptomatology distress. High internal consistency has been demonstrated for the depression $(\alpha=.94)$, anxiety $(\alpha=.87)$ and stress $(\alpha=.91)$ scales and validity. ${ }^{64-67}$

The Repetitive Thinking Questionnaire $\left(\mathrm{RTQ}^{32}\right)$; will be used to assess all types of repetitive negative thinking. The 31-item measure of transdiagnostic perseverative thinking comprises two subscales: repetitive negative thinking (27 items) and absence of repetitive thinking (4 items). It is rated on a five-point Likert scale ranging from 1 (not at all true) to 5 (very true). The RTQ has been found to have high internal consistency $(\mathrm{a}=0.72$ 0.93 ) and convergent validity. ${ }^{32}$

The Utrecht Grief Rumination Scale $\left(\mathrm{UGRS}^{27}\right)$; will be used to measure grief-specific rumination. This 15 -item self-report measure of recurrent, repetitive and selffocused thoughts about the loss comprises five subscales: thoughts about consequences and meaning of the loss; thoughts about social support; 'what-if' questions; 'why' questions and thoughts about feelings. A total score can be obtained by summing the subscales. Items are rated on a five-point Likert scale ranging from 1 (never) to 5 (very often). The UGRS has been found to have excellent internal consistency $(\alpha=.90)$, and validity. ${ }^{27}$

The Metacognitions Questionnaire-30 (MCQ-30 ${ }^{68}$ ); will be used to measure metacognitions. Each item on this 30 -item self-report measure is rated on a four-point Likert scale ranging from 1 (do not agree) to 4 (agree very much) on five separate subscales: positive beliefs about worry, negative beliefs about worry concerning uncontrollability and danger, low cognitive confidence, need to control thoughts, and cognitive selfconsciousness. ${ }^{68}$ A total MCQ-30 score is obtained by summing the subscales. A high score indicates stronger beliefs about the need to control and the negative consequences associated with intrusive thoughts. Internal consistency across individual subscales on the MCQ-30 
has been found to range from 0.72 to 0.93 with a total internal consistency of 0.93 and a test-retest reliability of $0.75 .^{35} 68$

The Quality of Life Enjoyment and Satisfaction Questionnaire-18 (Q-LES-Q-18 ${ }^{69}$ ); will be used to measure general quality of life and specific life domain levels of enjoyment and satisfaction. It is an 18-item self-report scale measuring specific life domains: physical health, subjective feelings, leisure time activity, social relationships, and satisfaction with medication. A five-point Likert scale ranging from 1 (not at all) to 5 (all the time) is used for responses. General quality of life index is measured by summing the 18 items. The Q-LES-Q-18 has good construct and concurrent validity, as well as high internal consistency ranging from 0.82 to 0.94 and test-retest reliability ranging from 0.71 to $0.83 .{ }^{69}$

\section{Therapist measure}

The Clinical Global Impression (CGI-Improvement Scale $\left(\right.$ CGI-IS $\left.^{70}\right)$; will be used to rate each participant's improvement or decline in post-treatment assessment. It is a global rating of improvement in clinical disorders ranging from 1 (very much improved) to 4 (no change) to 7 (very much worse). It has shown high inter-rater reliability $(0.87-0.99)$. To limit experimenter effects, the

Figure 1 The 'Metacognitive Grief Therapy (MCGT) Program' proposed design and progress phases. first author and an independent experienced psychologist will use this scale to compare baseline and follow-up interviews. Treatment response will be indicated by a rating of 1 (completely recovered), 2 (very much better) or 3 (much better). Inter-rater reliability will be calculated using Cohen's $\kappa$ statistic. ${ }^{71}$

\section{Trial design}

A randomised experimental pretest/posttest wait-list control group design with a 3-month and 6-month follow-up for the intervention group will be used to evaluate the effects of MCGT on PGD. The outcome variables are anxiety, stress and depression (DASS-21); grief distress (PG-13/CBI); rumination (RTQ/UGRS), metacognitions (MCQ-30) and quality of life (Q-LES-Q18). The independent variables are the group condition (MCGT; WL), and time (pretest, posttest, 3-month and 6-month follow-up). A study design flow chart is shown in figure 1 .

\section{Procedure}

Following ethical approval from Curtin University Human Research Ethics committee, participants who express an interest to take part in the study will be screened by telephone. Those who meet inclusion

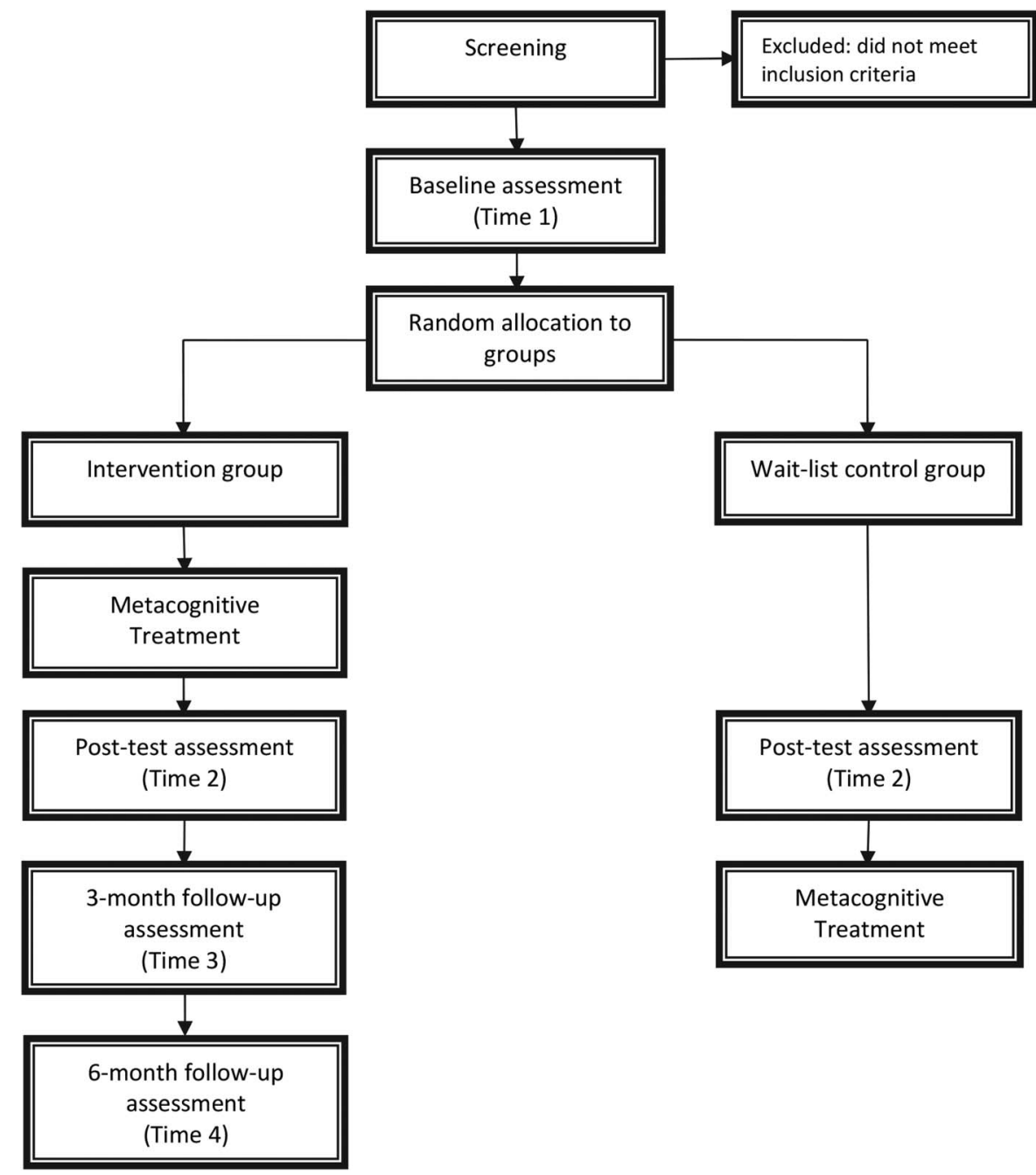


criteria will then attend an individual assessment session where they will read and sign consent forms, complete the MCMI and be interviewed using the MINI. Eligible participants will then be randomly allocated to the intervention or wait-list control condition via computer generated randomised numbers. The self-report measures will be conducted at preintervention and thereafter post-tests will be conducted at treatment completion, and at 3-months and 6-months from treatment completion for the intervention group only (via post or assistance from a psychology graduate trainee). Only the primary researcher will have access to the participants' data, which will be coded with an ID number and cross referenced in a university database to maintain confidentiality and preserve anonymity beyond the primary researcher. Any identifying information (ie, consent forms and demographic details) will be kept separate from the data and locked in a secured university filing cabinet for a period of 5 years, after which it will be destroyed. Participant compensation will be in the form of a gift voucher at post-test and 6-month assessment points. Following the post-test, MGCT will be offered to the wait-list control group, to ensure they also benefit from participation in the study. Participants in the waitlist control groups will receive a monthly phone call or email to ensure waiting for treatment is not detrimental and that they remain in the study. Treatment participants wishing to drop out of the study and wait-list participants demonstrating a need for immediate intervention will be withdrawn from the study and provided appropriate referral information.

\section{Data analysis}

A GLMM will be used to test H1 and H2. The GLMM is 'generalised' in the sense that it can handle outcome variables with markedly non-normal distributions; GLMM is 'mixed' in the sense that it includes random and fixed effects. The present GLMM includes one categorical random effect (participant), one categorical fixed effect (group: intervention, control), one ordinal fixed effect (time: pretest, post-test, 3-month follow-up, 6-month follow-up), and the Group ×Time interaction. A second GLMM will be developed to test H3. This GLMM will include one categorical random effect (participant) and one ordinal fixed effect (time: pretest, post-test, 3-month follow-up, 6-month follow-up) will be implemented through SPSS's (V.22) GENLINMIXED procedure. In order to optimise the likelihood of convergence, a separate GLMM analysis will be run for each of the outcome measures (PG-13, CBI, DASS, RTQ, UGRS, MCQ-30, Q-LES-Q-18). Analysing each outcome independently of the others will inflate the family-wise error rate and the per-test $\alpha$ will therefore need to be corrected to control the inflation. In order to conserve statistical power, the $\alpha$ correction will be applied within groups of conceptually related outcomes rather than across the entire set of outcomes.
GLMM will assume a normal probability distribution for the outcome and link it to the fixed effects (group, time, group $\times$ time) with an identity function. ${ }^{72}$ If the outcome does not have a normal distribution, then the parameter estimates of the covariance matrix will be computed with robust statistics. In order to make the model robust to violations of sphericity, the covariance matrix will be changed from the default of compound symmetry to autoregressive.

The mediation effects predicted in $\mathrm{H} 4$ will be tested with structural equation modelling in order to adjust for the relatively low reliabilities associated with change scores. The significance of the mediation pathways will be tested with a bootstrapping procedure based on 1000 draws as implemented by Mplus (V.5.2). Assuming 'moderate to large' mediation effects as per previous MCT intervention studies, ${ }^{40}{ }^{59} \mathrm{G} \times$ Power $3^{73}$ estimates that 34 participants will sufficiently power these tests. The mediation model will include one binary independent variable (group: intervention vs control), inputting to two mediating variables (metacognition and rumination), which then input to one outcome variable (eg, PGD). To conserve statistical power, separate mediation models will be tested for each of the other outcome variables. The mediating variables will be pre-post change scores, and the outcome variables will be pre-follow-up change scores.

Randomisation to groups should ensure that the groups are matched in terms of potentially confounding demographic covariates; but this process can be undermined by participant attrition. We will therefore attempt to identify confounding demographic variables (ie, demographic variables that show between-group differences and are correlated with the outcome at any of the assessment points) and control for them statistically by including them as covariates in the GLMM analyses. Pretest differences on the outcome variables are controlled by including pretest scores as the first level of the time factor in the GLMM analyses.

Participant attrition occurs in longitudinal data irrespective of the number of sessions completed. Participants who miss two treatment sessions will be regarded as dropouts. To optimise power, the outcome data for all participants will be analysed (regardless of the number of sessions completed) using the 'dose' (ie, number of sessions) as a covariate in the GLMM. Compared to the traditional statistical procedures for analysing behavioural change (eg, repeated measures analysis of variance), GLMM is less sensitive to participant attrition, does not rely on participants providing data at every assessment point, can deal with unequally spaced data collection points, is robust to unequal group sizes, does not require equal variances at each time point, does not require equal covariance between all pairs of time points (ie, sphericity), and is able to account for correlations among repeated measurements. ${ }^{72}$ GLMM will analyse all pretest scores on the outcomes regardless of whether participants attend posttest and follow-up 
assessments. This reduces sampling bias and the need to replace missing data. To assess if dropouts differ with completers they will be compared on demographics and baseline outcome measures. Treatment effect sizes on all outcomes will be calculated using Cohen's d statistic. ${ }^{74}$ Once an intervention effect has been established at the group level, an estimate of the clinical significance of the change will be calculated using the reliable change index. This will be assessed by calculating the degree to which participants change on the outcome variable divided by the $\mathrm{SE}$ of the difference between the pretest and posttest scores. A Reliable Change Index greater than an absolute value of 1.96 will reflect a reliable change. ${ }^{75}$

\section{Phase 3}

Programme integrity and content compliance

The first author and the group co-facilitator (both clinical psychology trainees) will be trained by a clinical psychologist MCT specialist. According to the intention-to-treat principle, researchers must assess protocol adherence in order to provide an unbiased assessment of treatment efficacy. ${ }^{73}$ To evaluate the integrity of the programme delivery and treatment adherence, each group session will be checked to ensure it is delivered in accordance with the MCGT session-by-session module overview. This involves the independent completion of a programme implementation effectiveness checklist by the first author and co-facilitator at the completion of each session. Each item will be rated using a 10-point Likert scale. A minimum of $10 \%$ of the video recordings for each session will also be reviewed by a supervisor with a clinical psychology background for protocol adherence. Inter-rater reliability will be calculated using the 'average measures' intraclass correlation using the 'two way random model' in conjunction with the 'consistency procedure'. ${ }^{76}$

\section{Social validity}

At programme completion, the social validity of the entire programme will be measured using the Programme Satisfaction Questionnaire adapted from a depression and anxiety prevention programme. ${ }^{77}$ The participants will complete questionnaires comprising an overall programme rating (section $\mathrm{A}$ ), the practicality of the skills taught (section $\mathrm{B}$ ) and a qualitative interview (section $\mathrm{C}$ ). Sections A and B will be rated on a five-point Likert scale ranging from 1 (not at all) to 5 (very much). The five open-ended questions in section $\mathrm{C}$ allow participants to identify programme components least and most enjoyed and information most utilised. The questionnaires will determine the appropriateness and acceptability of the programme. Descriptive statistics will be used to analyse the quantitative responses of the Programme Satisfaction Questionnaire. The open-ended qualitative responses to each question will be summarised.

\section{DISCUSSION}

There is very robust evidence demonstrating that PGD differs from normal grief in terms of symptoms, aetiology, outcomes, course and response to treatment ${ }^{4}$ and that people with PGD experience self-blame ${ }^{78}$ and are under-represented in mental health services. ${ }^{5}$ Existing interventions show some promise, but their effectiveness is limited. Recent studies suggest there is a need for treatments to incorporate techniques that deal with rumination, as it is associated with and predictive of PGD. ${ }^{27} 79$ Given the severity of PGD, it is important to address directly the factors involved to limit the extent to which the death of a significant other may constitute a broader risk to bereaved individuals' long-term health outcomes.

This paper is a comprehensive description of the methodology used to develop, implement and evaluate MCGT, a MCT programme for PGD. MCT is an efficacious treatment for several psychological disorders (supporting the regulation of cognitive processes); however, no research has been conducted on the effectiveness of this treatment for people with PGD. It is proposed that an intervention based on the metacognitions of this subset of bereaved individuals will target the dysfunctional coping processes (eg, rumination, worry and avoidance behaviours) that keep attention focused on distressing and unhelpful information, depleting the processing resources required for thinking to return to its normal trauma free status.

The proposed study will evaluate the efficacy of manualised MCGT to reduce PGD symptoms. This study will determine if MCGT is likely to be effective in reducing PGD symptoms and will establish the mechanisms by which the intervention may be effective. The study will also determine if MCGT is able to enhance the quality of life for people with PGD and if the intervention is acceptable to them. IF MCGT is not effective, the study will identify the factors that contributed to a negative outcome.

This research has implications for healthcare professionals who work with bereaved clients. A validated short intervention that is acceptable to bereaved people would benefit services where long-term care is not an alternative. ${ }^{6}$ The proposed research is innovative because it will provide much needed empirical evidence to guide future programmes that target PGD and, if MCGT proves to be effective, it will provide a precise intervention that ameliorates PGD symptomatology. As such, this study makes an important contribution to theory and practice.

Dissemination The results will be documented in published peer reviewed academic journals. No identifying information will be provided; participant confidentiality will be upheld at all times.

Contributors LJB conceived the study. JW, MO'C, LJB, CSR developed the research design, methodology, intervention and implementation. JW drafted the manuscript. CSR provided clinical expertise and MCT training. RTK provided statistical input into the design. All authors edited drafts of the manuscript and approved the final manuscript. 
Funding JW is a Doctor of Philosophy (Clinical Psychology) candidate and received Faculty funding for the manuscript's preparation. LJB is supported by the Australian Research Council (DE120101640). Contact Name: Jenine Wenn, Curtin University, Perth, Western Australia, Australia, (+618)9266 7279, jenine.wenn@postgrad.curtin.edu.au

Competing interests None declared.

Ethics approval Ethics approval gained from Curtin University.

Provenance and peer review Not commissioned; externally peer reviewed.

Data sharing statement All de-identified aggregate data for these studies will be freely available in the published papers. All quantitative data generated from the project will be made available via Curtin Universities Research Data Repository (espace@Curtin). DOIs to these data will be provided and cited in any future published articles using this data. There are no security, licensing or ethical issues related to the expected data. No pre-existing data will be used, all data will be generated directly as a result of the project.

Open Access This is an Open Access article distributed in accordance with the Creative Commons Attribution Non Commercial (CC BY-NC 4.0) license which permits others to distribute, remix, adapt, build upon this work noncommercially, and license their derivative works on different terms, provided the original work is properly cited and the use is non-commercial. See: http:// creativecommons.org/licenses/by-nc/4.0/

\section{REFERENCES}

1. Horowitz MJ, Siegel B, Holen A, et al. Diagnostic criteria for complicated grief disorder. Am J Psychiatry 1997;154:904-10.

2. Lichtenthal WG, Cruessa DG, Prigerson H. A case for establishing complicated grief as a distinct mental disorder in DSM-V. Clin Psychol Rev 2004;24:637-62.

3. Prigerson HG, Vanderwerker LC, Maciejewski PK. A case for inclusion of prolonged grief disorder in DSM-V. In: Stroebe MS, et al., ed. Handbook of bereavement research and practice: advances in theory and intervention, Washington DC: American Psychological Association Press, 2008:165-86.

4. Prigerson HG, Horowitz MJ, Jacobs SC, et al. Prolonged grief disorder: psychometric validation of a criteria proposed for DSM-V and ICD-11. PLoS Med 2009;6:e1000121.

5. Lichtenthal WG, Nilsson M, Kissane DW, et al. Underutilization of mental health services among bereaved caregivers with prolonged grief disorder. Psychiatr Serv 2011;62:1225-9.

6. Kiesler $\mathrm{C}$. The next wave of change for psychology and mental health services in the health care revolution. Am Psychol 2000;55:481-7.

7. Lund D, Caserta M, Utz R, et al. Experiences and early coping of bereaved spouses/partners in an intervention based on the dual process model. Omega 2010;61:291-313.

8. American Psychiatric Association. Diagnostic and statistical manual of mental disorders: DSM-5. 5th edn. Washington DC: American Psychiatric Press, 2013.

9. Maercker A, Brewin CR, Bryant RA, et al. Proposals for mental disorders specifically associated with stress in the ICD-11. Lancet 2013;381:1683-5.

10. Breen LJ, O'connor M. The fundamental paradox in the grief literature: a critical reflection. Omega 2007;55:199-218.

11. Payne S, Jarrett N, Wiles $\mathrm{R}$, et al. Counselling strategies for bereaved people offered in primary care. Couns $P$ sychol $Q$ 2002;15:161-77.

12. Breen LJ. Professionals' experiences of grief counselling implications for bridging the gap between research and practice. Omega 2011;62:285-303.

13. Currier JM, Neimeyer RA, Berman JS. The effectiveness of psychotherapeutic interventions for bereaved persons a comprehensive quantitative review. Psychol Bull 2008;134:648-61.

14. Reynolds CF, Miller MD, Pasternak RE, et al. Treatment of bereavement-related major depressive episodes in later life: a randomized, double-blind, placebo controlled study of acute and continuation treatment with nortriptyline and interpersonal psychotherapy. Am J Psychiatry 1999;156:202-8.

15. Zisook S, Shuchter SR, Pedrelli P. Acute, open trial of bupropion SR therapy in bereavement. J Clin Psychiatry 2001;62:227-30.

16. Bryant RA, Kenny L, Joscelyne A et al. Treating prolonged grief disorder a randomized clinical trial. JAMA Psychiatry 2014;71:1332-9.

17. Boelen PA, De Keijser J, Van Den Hout MA et al. Treatment of complicated grief: a comparison between cognitive-behavioral therapy and supportive counseling. J Consult Clin Psychol 2007;75:277-84.

18. Shear K, Frank E, Houck PR, et al. Treatment of complicated grief: a randomized controlled trial. JAMA 2005;293:2601-8.

19. Wittouck C, Van Autreve S, De Jaegere E, et al. The prevention and treatment of complicated grief: a meta-analysis. Clin Psychol Rev 2011;31:69-78.

20. Boelen PA, Van Den Hout MA, Van Den Bout J. A cognitivebehavioral conceptualization of complicated grief. Clin Psychol 2006;13:109-28.

21. Stroebe MS, Hansson RO, Schut $\mathrm{H}$, et al. Handbook of bereavement research and practice: advances in theory and intervention. Washington DC: American Psychological Association, 2008.

22. Wells A, Matthews G. Attention and emotion: a clinical perspective. Hove, UK: Lawrence Erlbaum \& Associates, 1994.

23. Holland JM, Currier JM, Neimeyer RA. Meaning reconstruction in the first two years of bereavement: the role of sense-making and benefit-finding. Omega 2006;53:175-91.

24. Field N, Gal-Oz E, Bonanno G. Continuing bonds and adjustment at 5 years after the death of a spouse. J Consult Clin Psychol 2003;71:110-17.

25. Richards TA, Acree M, Folkman S. Spiritual aspects of loss among partners of men with AIDS: postbereavement follow-up. Death Stud 1999;23:105-27.

26. Boelen PA, Stroebe MS, Schut HAW, et al. Continuing bonds and grief: a prospective analysis. Death Stud 2006;30:767-76.

27. Eisma MC, Stroebe MS, Schut HAW, et al. Development and psychometric evaluation of the Utrecht Grief Rumination Scale. J Psychopathol Behav Assess 2014;36:165-76.

28. Stroebe M, Boelen PA, Van Den Hout M, et al. Ruminative coping as avoidance: a reinterpretation of its function in adjustment to bereavement. Eur Arch Psychiatry Clin Neurosci 2007;257:462-72.

29. Eisma MC, Rinck M, Stroebe MS, et al. Rumination and implicit avoidance following bereavement: an approach avoidance task investigation. J Behav Ther Exp Psychiatry 2015;47:84-91.

30. Ehring T, Watkins ER. Repetitive negative thinking as a transdiagnostic process. Int J Cogn Ther 2008;1:192-205.

31. Mcevoy PM, Watson $\mathrm{H}$, Watkins ER, et al. The relationship between worry, rumination, and comorbidity: evidence for repetitive negative thinking as a transdiagnostic construct. J Affect Disord 2013;151:313-20.

32. Mcevoy PM, Mahoney A, Moulds ML. Are worry, rumination, and post-event processing one and the same? Development of the repetitive thinking questionnaire. J Anxiety Disord 2010;24:509-19.

33. Nolen-Hoeksema S, Wisco BE, Lyubomirsky S. Rethinking rumination. Perspect Psychol Sci 2008;3:400-24.

34. Eisma MC, Schut HAW, Stroebe MS, et al. Adaptive and maladaptive rumination after loss: a three-wave longitudinal study. Br J Clin Psychol 2015;54:163-80.

35. Van Der Houwen K, Stroebe M, Schut H, et al. Mediating processes in bereavement: the role of rumination, threatening grief interpretations, and deliberate grief avoidance. Soc Sci Med 2010;71:1669-76.

36. Wells A. Metacognitive therapy for anxiety and depression. New York: The Guilford Press, 2009.

37. Stroebe MS, Schut $\mathrm{H}$. The dual process model of coping with bereavement: rationale and description. Death Stud 1999;23:197.

38. Nordahl HM. Effectiveness of brief Metacognitive Therapy versus Cognitive-Behavioral Therapy in a general outpatient setting. Int $J$ Cogn Ther 2009;2:152-9.

39. Siegle GJ, Ghinassi F, Thase ME. Neurobehavioral therapies in the 21st century: summary of an emerging field and an extended example of cognitive control training for depression. Cogn Ther Res 2007;31:235-62.

40. Wells A, Fisher P, Myers S, et al. Metacognitive therapy in treatment resistant depression: a platform trial. Behav Res Ther 2012;50:367.

41. Fisher PL, Wells A. Metacognitive therapy for obsessive-compulsive disorder: a case series. J Behav Ther Exp Psychiatry 2008;39:117-32.

42. Moritz S, Jelinek L, Hauschildt $M$, et al. How to treat the untreated: effectiveness of a self-help metacognitive training program (myMCT) for obsessive-compulsive disorder. Dialogues Clin Neurosci 2010;12:209-20.

43. Rees CS, Van Koesveld KE. Metacognitive therapy treatment manual: group and individual protocol. In: Rees CS, ed. Obsessivecompulsive disorder. Melbourne: IP Communications, 2009.

44. Mcevoy PM, Perinib SJ. Cognitive behavioral group therapy for social phobia with or without attention training: a controlled trial. $J$ Anxiety Disord 2009;23:519-28.

45. Mcevoy PM, Mahoney A, Perini SJ, et al. Changes in post-event processing and metacognitions during cognitive behavioral group therapy for social phobia. J Anxiety Disord 2009;23:617-23. 
46. Van Der Heiden C, Muris P, Van Der Molen HT. Randomized controlled trial on the effectiveness of metacognitive therapy and intolerance-of-uncertainty therapy for generalized anxiety disorder. Behav Res Ther 2012;50:100-9.

47. Wells $\mathrm{A}$, Welford $\mathrm{M}$, King $\mathrm{P}$, et al. A pilot randomized trial of metacognitive therapy vs applied relaxation in the treatment of adults with generalized anxiety disorder. Behav Res Ther 2010;48:429-34

48. Wells A, Colbear JS. Treating posttraumatic stress disorder with metacognitive therapy: a preliminary controlled trial. J Clin Psychol 2012;68:373-81.

49. Wells A, Sembi S. Metacognitive therapy for PTSD: a preliminary investigation of a new brief treatment. J Behav Ther Exp Psychiatry 2004;35:307-18.

50. Wells A, Welford M, Fraser J, et al. Chronic PTSD treated with metacognitive therapy: an open trial. Cogn Behav Pract 2008;15:85-92

51. Marshall M. Sampling for qualitative research. Fam Pract 1996;13:522-6.

52. Tomarken A, Roth $\mathrm{A}$, Holland $\mathrm{J}$, et al. Examining the role of trauma, personality, and meaning in young prolonged grievers.

Psychooncology 2012;21:771-7.

53. Lincoln YS, Guba E. Naturalistic inquiry. Thousand Oaks, CA: SAGE Publications, 1985

54. Chambless DL, Ollendick TH. Empirically supported psychological interventions: controversies and evidence. Annu Rev Psychol 2001:52:685-716.

55. Jané-Llopis E, Hosman C, Jenkins R, et al. Predictors of efficacy in depression prevention programmes: meta-analysis. $\mathrm{Br} J$ Psychiatry 2003;183:384-97.

56. Sheehan DV, Lecrubier Y, Harnett Sheehan K, et al. The validity of the Mini International Neuropsychiatric Interview (MINI) according to the SCID-P and its reliability. Eur Psychiatry 1997;12:232-41.

57. Millon T. Millon Clinical Multiaxial Inventory manual. 3rd edn New York: Holt, Rinehart, \& Winston, 1983.

58. Faul F, Erdfelder E, Lang A-G, et al. A flexible statistical power analysis program for the social behavioral, and biomedical sciences. Behav Res Methods 2007;39:175-91.

59. Wells A, King P. Metacognitive therapy for generalized anxiety disorder: an open trial. J Behav Ther Exp Psychiatry 2006;37:206-12.

60. Overholser JC. Retest reliability of the Million Clinical Multiaxial Inventory. J Pers Assess 1990;55:202-7.

61. Lecrubier $Y$, Sheehan DV, Weiller E, et al. The Mini International Neuropsychiatric Interview (MINI). A short diagnostic structured interview: reliability and validity according the the CIDI. Eur Psychiatry 1997;12:224-31.

62. Burnett $P$, Middleton BR, Martinek N. Measuring core bereavement phenomena. Psychol Med 1997;27:49-57.

63. Keesee NJ, Currier JM, Neimeyer RA. Predictors of grief following the death of one's child: the contribution of finding meaning. $J$ Clin Psychol 2008;64:1145-63.
64. Lovibond $\mathrm{P}$, Lovibond $\mathrm{S}$. The structure of negative emotional states: comparison of the Depression Anxiety Stress Scales (DASS) with the Beck Depression and Anxiety Inventories. Behav Res Ther 1995;33:335-43.

65. Taylor R, Lovibond PF, Nicholas MK, et al. The utility of somatic items in the assessment of depression in chronic pain patients: a comparison of the Zung Self-rating Depression Scale (SDS) and the Depression Anxiety Stress Scales (DASS) in chronic pain and clinical and community samples. Clin J Pain 2005;21:91-100.

66. Szabó M. The short version of the Depression Anxiety Stress Scales (DASS-21): factor structure in a young adolescent sample.

$J$ Adolesc 2010;33:1-8.

67. Antony M, Bieling PJ, Cox BJ, et al. Psychometric properties of the 42-item and 21-item versions of the depression anxiety stress scales in clinical groups and a community sample. Psychol Assess 1998; 10:176-81.

68. Wells A, Cartwright-Hatton S. A short form of the metacognitions questionnaire: properties of the MCQ-30. Behav Res Ther 2004;42:385-96.

69. Ritsner M, Kurs R, Gibel A, et al. Validity of an abbreviated Quality of Life Enjoyment and Satisfaction Questionnaire (Q-LES-Q-18) for schizophrenia, schizoaffective, and mood disorder patients. Qual Life Res 2005;14:1693-703.

70. Guy W. ECDEU assessment manual for psychopharmacology. Rockville, MD: US Department of Heath, Education, and Welfare Public Health Service Alcohol, Drug Abuse, and Mental Health Administration, 1976.

71. Cohen J. A coefficient for agreement for nominal scales. Educ Psychol Meas 1960;20:37-46.

72. Mcculloch CE, Neuhaus JM. Generalized linear mixed models in encyclopedia of environmetrics. John Wiley \& Sons, Ltd: Online, 2013.

73. Montori VM, Guyatt GH. Intention-to-treat principle. CMAJ 2001;165:1339-41.

74. Durlak JA. How to select, calculate, and interpret effect sizes. J Pediatr Psychol 2009;34:917-28.

75. Jacobson N, Truax P. Clinical significance: a statistical approach to defining meaningful change in psychotherapy research. $J$ Consult Clin Psychol 1991;59:12-19.

76. Yen M, Lo L. Examining test-retest reliability an intra-class correlation approach. Nurs Res 2002;51:59-62.

77. Roberts C, Ballantyne F, Van Der Klift P. Aussie optimism. Social life skills. Teacher resource. Perth, Australia: Curtin University of Technology, 2003.

78. Johnson JG, First MB, Block S, et al. Stigmatization and receptivity to mental health services among recently bereaved adults. Death Stud 2009:33:691-711.

79. Boelen PA, Van Den Bout J, Van Den Hout MA. The role of negative interpretations of grief reactions in emotional problems after bereavement. J Behav Ther Exp Psychiatry 2003;34:225-38. 